\title{
Tales of the Unexpected: Selectivity of Nucleophilic Additions to Zigzag Nanographene Diones ${ }^{\text {II }}$
}

Peter Ribar, ${ }^{\text {ab }}$ Leoš Valenta, ${ }^{\text {ac }}$ Tomáš Šolomek, ${ }^{* b d}$ and Michal Juríček*d

${ }^{a}$ These authors contributed equally to this work.

${ }^{b}$ Department of Chemistry, University of Basel, St. Johanns-Ring 19, CH-4056 Basel, Switzerland

'Department of Chemistry, University of Zurich, Winterthurerstrasse 190, CH-8057 Zurich, Switzerland

dPrievidza Chemical Society, M. Hodžu 10/16, 97101 Prievidza, Slovak Republic

IThe title refers to Roald Dahl's book Tales of the Unexpected.

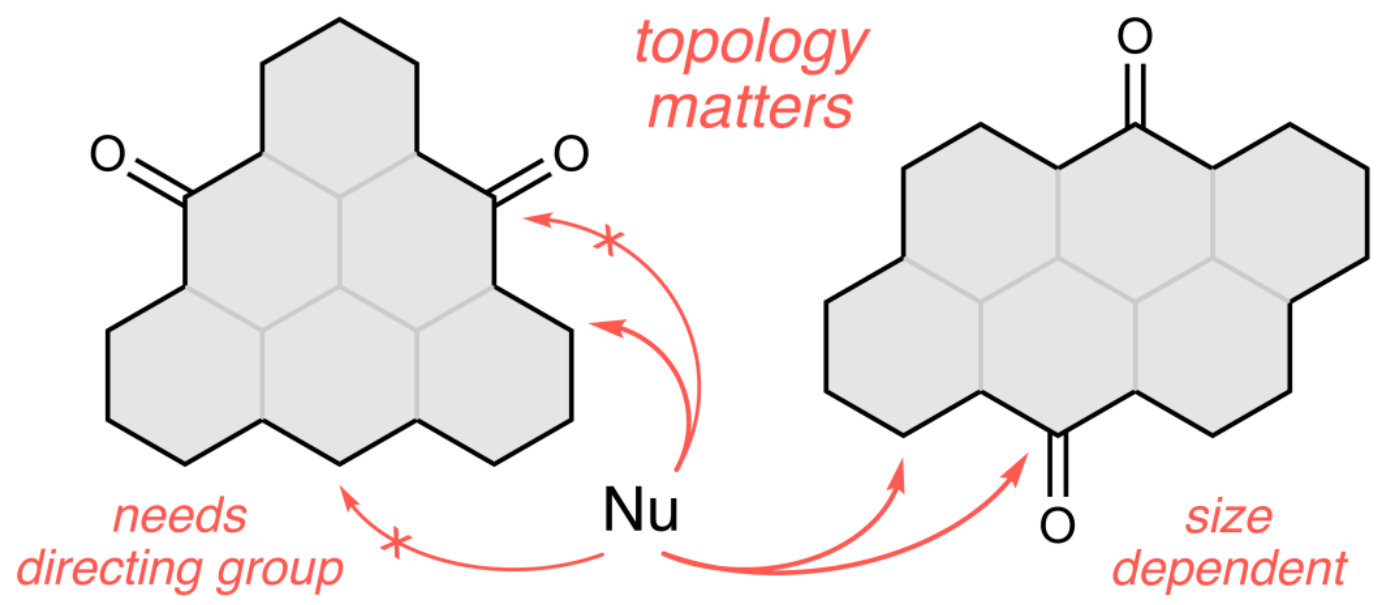

Abstract: Nucleophilic addition of carbon-centered nucleophiles to nanographene ketones represents a valuable late-stage method for the functionalization of zigzag nanographenes, but its use is rare in the chemical literature. Using two model systems, non-Kekulé triangulene-4,8-dione and Kekulé anthanthrone, we identify unexpected regioselectivities and uncover the rules that govern these reactions. Considering the large number of nanographene ketones that have been reported since the pioneering work of Eric Clar, this method enables synthesis and exploration of hitherto unknown functionalized nanographenes. 


\section{Introduction}

The finite fragments of graphene — nanographenes — are ideal molecular models for investigating the relationship between the structure and properties of semiconducting ${ }^{1}$ and magnetic ${ }^{2}$ materials based on graphene nanoribbons. The bottom-up synthetic access to these materials ${ }^{3}$ is key for meeting the expectations and shaping the future of the molecular electronics. ${ }^{4}$ Routine issues that need to be addressed when synthesizing nanographenes are stability ${ }^{5}$ and solubility. ${ }^{6}$ A classic example are acenes, ${ }^{7}$ the thinnest possible nanoribbons, elongation of which increases charge mobility but decreases chemical stability. ${ }^{8}$ Lateral extension (see anthracene and anthanthrene in Figure 1) overcomes this problem but limits the solubility, and thus processability, of such nanographenes. ${ }^{9}$ Conveniently, both issues can be solved by installment of peripheral substituents that prevent molecules from reacting and stacking, ${ }^{10}$ in addition to tuning their molecular ${ }^{11}$ and bulk properties. ${ }^{12}$ The drawback is that introduction of substituents embodies an additional hurdle on the synthetic pathway and may defy synthetic chemists to reach the target.

Thanks to the recent advances in $\mathrm{C}-\mathrm{H}$ activation, the cross-coup-ling reactions enable introduction of substituents to different positions. ${ }^{13}$ This method, however, cannot always be applied to all positions in zigzag nanographenes. For example, it would be difficult, if possible at all, to modify positions at the center of the edges in Clar's hydrocarbon triangulene (Figure 1). A common approach to install substituents in zigzag nanographenes is a nucleophilic addition to an aldehyde prior to the nanographene core formation by the Friedel-Crafts alkylation, ${ }^{14}$ which decreases modularity. A better but far less common method is to add nucleophiles to the products of the Friedel-Crafts acylation, the simplest method to construct zigzag nanographenes. ${ }^{15}$

This strategy has been successful for making a variety of acenes up to pentacene by 1,2-additions of carbon-centered nucleophiles to the corresponding quinones (Figure 1), ${ }^{12,16}$ while only acetylide 1,2-additions have been reported for hexacene and higher acene quinones. ${ }^{10 b, 17}$ On lateral extension, acetylide 1,2-additions are common, ${ }^{18}$ whereas only one example of heteroaryl 1,2addition has been described on anthanthrone. ${ }^{18 a}$ Other aryl substituents have been introduced by multistep sequences. ${ }^{14,19}$ Despite the examples, where 1,2-additions work, it is striking that this strategy is not generally applied to extended systems, for which the ketone precursors are known. For instance, triangulene (Figure 1) is an example of an open-shell zigzag nanographene that has never been isolated in the solid state. 


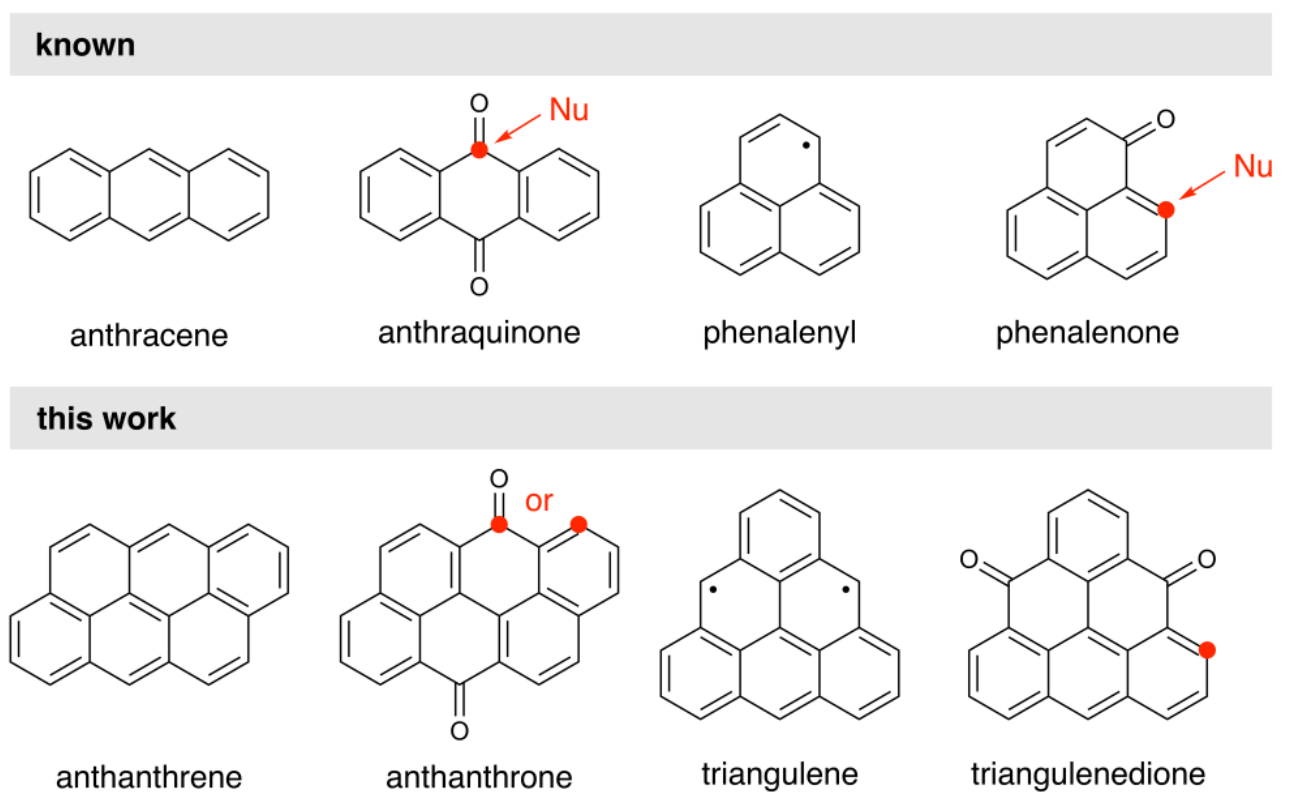

Figure 1. Regioselectivity of additions of carbon-centered nucleophiles to ketone precursors of Kekulé (anthracene, anthanthrene) and non-Kekulé (phenalenyl ${ }^{20}$, triangulene) nanographenes.

This non-Kekulé triplet diradical was only made twice in its neutral form: in 2001 as a short-lived tri-tert-butyl derivative ${ }^{5 a}$ and in 2017 as a naked system on surface under ultra-high vacuum. ${ }^{21}$ It is a paradox that for almost 70 years, the ideal precursor for such task, triagulene-4,8-dione, is known since the first synthetic attempts by Eric Clar in $1950 \mathrm{~s}^{22}$ All it would take to make persistent triangulene is to perform a nucleophilic 1,2-addition at the carbonyl centers of the triagulenedione core and reduce the ensuing diol. Where is the catch?

We selected two isomeric zigzag model systems, based either on triangulene or anthanthrene (Figure 1), to uncover the rules that govern nucleophilic additions on zigzag nanographene ketones and to understand why the use of this method is uncommon in the chemical literature.

\section{Results}

Each model system comprises of 22 carbon atoms and six benzenoid rings. While the Kekulé conjugation topology of anthanthrene is the same as that of acenes, the isomeric triangulene has a non-Kekulé topology. We improved the solubility of model diones by a minimum number of substituents distant from the reactive centers (see the SI) to get a better control over the reactions. We used two readily available reagents as carbon-centered nucleophiles, phenylethynyllithium (PhCCLi) and 3,5-di-tert-butyl-phenylmagnesium bromide ( $\mathrm{ArMgBr})$, which serve as models for 
common substituents that control solubility, aggregation, electronic structure, and solid-state morphology of nanographenes. It was important to determine the exact concentration of the reagent before each experiment to get reproducible results. In the case of double 1,2-additions, a reduction of the diol intermediate by $\mathrm{SnCl}_{2}$ was performed to obtain the corresponding nanographene, which simplified purification and product identification. Likewise, conjugate additions were followed by an oxidation of the enol intermediate by $\mathrm{O}_{2}$ or $\mathrm{I}_{2}$. The structure of all products was confirmed by 2D NMR spectroscopy to unambiguously identify the position, where the nucleophilic addition took place.

\subsection{Triangulenedione}

We attempted a double 1,2-addition to triangulenedione by reacting its soluble derivative T1 (Ar =3,5-di-tert-butylphenyl, Scheme 1) with an excess of ArMgBr. If successful, the product would yield an ideal precursor of a persistent triangulene. Instead, we obtained a complex mixture of products, which we were unable to identify. One equivalent of $\mathrm{ArMgBr}$, however, gave a clean 1,4-addition providing T2 after oxidation in 75\% yield (Scheme 1). A more reactive ArLi nucleophile expected to favor a 1,2-addition preserved the 1,4-selectivity, albeit with a lower yield (44\%). To our surprise, we observed no conversion in the reaction of dione $\mathbf{T} 1$ with one equivalent of $\mathrm{PhCCLi}$, an excess led again to a complex reaction mixture. This reactivity is in stark contrast to that observed for acene quinones, which give exclusively 1,2-additions. The most closely related system to dione $\mathbf{T 1}$ that is known to undergo 1,4-additions is phenalenone ${ }^{23}$ (Figure 1), even though it has only one carbonyl group. Nevertheless, its lowest unoccupied molecular orbital (LUMO, Figure S1) is analogous to that of triangulenedione, which suggests that conjugation topology ${ }^{20}$ governs the selectivity.

Scheme 1. Nucleophilic additions to triangulenedione ${ }^{a}$

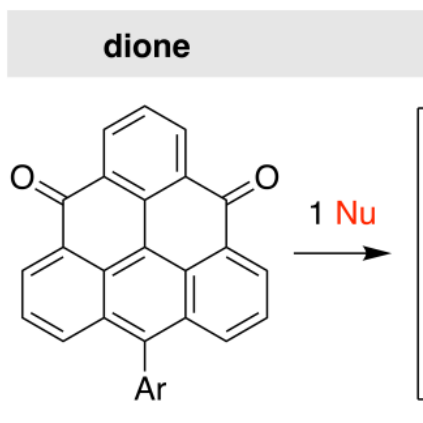

T1 1,4-addition

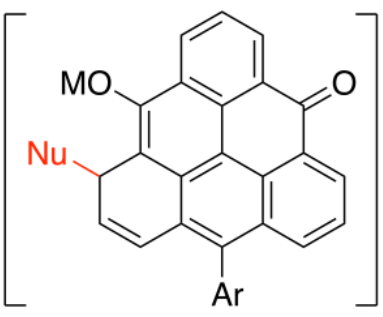

${ }^{a} \mathrm{Ar}=\mathrm{Nu}=3,5$-di-tert-butylphenyl, $\mathrm{M}=\mathrm{MgBr}$ or Li. oxidation<smiles></smiles>

T2 


\subsection{Anthanthrone}

This dione is a lateral extension of anthraquinone. It displays analogous LUMO (Figure S1) and one would expect it to undergo solely 1,2-additions, like anthraquinone. Indeed, the reaction of dione $\mathbf{A 1}$ ( $\mathrm{R}=3$,5-di-tert-butylphenyl) with two equivalents of PhCCLi gives the product of a double 1,2-addition (A2; 72\% yield); the use of one equivalent results in 1,2-product $\mathbf{A 3}$ (86\%, Scheme 2 and Table 1). The reaction with an excess of $\mathrm{ArMgBr}$, however, leads to a product of consecutive 1,2- and 1,4-additions (A4; 83\%).

Scheme 2. Nucleophilic additions to anthanthrone ${ }^{a}$<smiles></smiles>

\section{1,2-addition ${ }^{b}$}<smiles>[R]C1=Cc2c3c4cccc3c2c2c(cc([R])c3cccc(c32)C1(O)O)c4=O</smiles>

A3, $\mathrm{R}=\mathrm{Ar}, \mathrm{Nu}=\mathrm{PhCC}$

A1, $\mathrm{R}=\mathrm{Ar}$

A8, $\mathrm{R}=\mathrm{OPhCC}$

A6, $\mathrm{R}=\mathrm{Nu}=\mathrm{Ar}$

\section{1,4-addition ${ }^{c}$}<smiles></smiles>

A5, $\mathrm{R}=\mathrm{Nu}=\mathrm{Ar}$ $\downarrow>2 \mathrm{Nu}$

$1,2 \& 1,2^{d}$<smiles></smiles>

A2, $\mathrm{R}=\mathrm{Ar}, \mathrm{Nu}=\mathrm{PhCC}$

A9, $\mathrm{R}=\mathrm{OPhCC}, \mathrm{Nu}=\mathrm{PhCC}$
$1,2 \& 1,4^{e}$<smiles>[R]c1cc2c(O)c3c(N)ccc4c([R])cc(c5cccc1-5)c(N)c2c43</smiles>

A4, $\mathrm{R}=\mathrm{Nu}=\mathrm{Ar}$

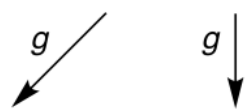

$1,4 \& 1,4^{c}$

A7, $\mathrm{R}=\mathrm{Nu}=\mathrm{Ar}$

A10, $\mathrm{R}=\mathrm{oPhCC}, \mathrm{Nu}=\mathrm{Ar}$

A11, $\mathrm{R}=\mathrm{Ar}, \mathrm{Nu}=\mathrm{Mes}$

${ }^{a} \mathrm{Ar}=3,5$-di-tert-butylphenyl, $\mathrm{PhCC}=$ phenylethynyl, oPhCC $=$ (4-octylphenyl)ethynyl, $\mathrm{Mes}=$ mesityl. ${ }^{b, c, d, e}$ Isolated after subsequent ${ }^{b}$ protonation, ${ }^{c}$ oxidation $\left(\mathrm{O}_{2}\right.$ or $\left.\mathrm{I}_{2}\right),{ }^{d}$ reduction $\left(\mathrm{SnCl}_{2}\right)$, and ${ }^{e}$ dehydration. ${ }^{f}$ Without protonation after the first 1,2 -addition. ${ }^{g}$ Without oxidation after the first 1,4-addition; nucleophile adds to the enol intermediate of 1,4-addition (Figure 4). 
Table 1. Nucleophilic additions to triangulenedione and anthanthrone ${ }^{a}$

\begin{tabular}{|c|c|c|c|c|}
\hline dione & $\mathrm{Nu}$ & $\begin{array}{l}\mathrm{Nu} \\
\text { equiv }\end{array}$ & $\begin{array}{l}\text { product } 1 \\
(\#, \text { yield) }\end{array}$ & $\begin{array}{l}\text { product } 2 \\
(\#, \text { yield })\end{array}$ \\
\hline T1 & $\mathrm{ArMgBr}$ & 1 & $\begin{array}{c}1,4 \\
(\mathbf{T} 2,75 \%)\end{array}$ & $-{ }^{b}$ \\
\hline T1 & ArLi & 1 & $\begin{array}{c}1,4 \\
(\mathbf{T} 2,44 \%)\end{array}$ & $-{ }^{b}$ \\
\hline T1 & $\begin{array}{l}\mathrm{ArLi}+ \\
\mathrm{DMPU}\end{array}$ & $1+2$ & $n . r^{c}$ & n.r. ${ }^{c}$ \\
\hline A1 & PhCCLi & 2 & $\begin{array}{c}1,2 \& 1,2 \\
(\mathbf{A} 2,72 \%)\end{array}$ & $-{ }^{b}$ \\
\hline A1 & PhCCLi & 1 & $\begin{array}{c}1,2 \\
(\mathbf{A} 3,86 \%)\end{array}$ & $-{ }^{b}$ \\
\hline A1 & $\mathrm{ArMgBr}$ & 10 & $\begin{array}{c}1,2 \& 1,4 \\
(\mathbf{A} 4,83 \%)\end{array}$ & $-{ }^{b}$ \\
\hline A1 & $\mathrm{ArMgBr}$ & 1 & $\begin{array}{c}1,4 \\
(\mathbf{A 5}, 50 \%)\end{array}$ & $\begin{array}{c}1,2 \\
(\mathbf{A 6}, 34 \%)\end{array}$ \\
\hline A1 & ArLi & 13 & $\begin{array}{c}1,2 \& 1,4 \\
(\mathbf{A} 4,75 \%)\end{array}$ & $\begin{array}{c}1,4 \& 1,4 \\
(\mathbf{A} 7,22 \%)\end{array}$ \\
\hline A1 & $\begin{array}{l}\text { ArLi }+ \\
\text { DMPU }\end{array}$ & $\begin{array}{l}10+ \\
20^{d}\end{array}$ & n.r. ${ }^{c}$ & $n . r .{ }^{c}$ \\
\hline A1 & MesMgBr & 10 & $\begin{array}{c}1,4 \& 1,4 \\
(\mathbf{A 1 1}, 91 \%)\end{array}$ & $-{ }^{b}$ \\
\hline A8 & PhCCLi & 10 & $\begin{array}{c}1,2 \& 1,2 \\
(\mathbf{A 9}, 86 \%)\end{array}$ & $-{ }^{b}$ \\
\hline A8 & $\mathrm{ArMgBr}$ & 10 & $\begin{array}{c}1,4 \& 1,4 \\
(\mathbf{A 1 0}, 69 \%)\end{array}$ & $-{ }^{b}$ \\
\hline A8 & $\mathrm{ArLi}$ & 2 & $\begin{array}{c}1,4 \& 1,4 \\
(\mathbf{A 1 0}, 40 \%)\end{array}$ & $-{ }^{b}$ \\
\hline A8 & $\begin{array}{l}\text { ArLi }+ \\
\text { DMPU }\end{array}$ & $2+4$ & n.r. ${ }^{c}$ & $n . r^{c}$ \\
\hline
\end{tabular}

${ }^{a}$ Refer to Schemes 1 and 2. A1, T1: $\mathrm{R}=\mathrm{Ar}=3,5$-di-tert-butylphenyl, A8: $\mathrm{R}=$ (4octylphenyl)ethynyl, Mes $=$ mesityl, DMPU $=N, N^{\prime}$-dimethylpropyleneurea. ${ }^{b}$ Not observed. ${ }^{c}$ No reaction. ${ }^{d}$ Quenching of the reagent with DMF led to $>90 \%$ ArCHO formation. 
To determine which addition is faster, we performed this reaction with one equivalent of $\mathrm{ArMgBr}$. Unexpectedly, we isolated the product of 1,4 -addition $(\mathbf{A 5} ; 50 \%)$ in a yield higher than that of 1,2 addition (A6; 34\%). To the best of our knowledge, this has never been observed in linear acene quinones. This result suggests that each initial adduct undergoes the subsequent addition with a complementary selectivity to form one product A4. We tested if a more reactive nucleophile such as ArLi would suppress 1,4-addition, but we observed an opposite effect. Reacting an excess of ArLi with A1, we isolated the product of a double 1,4-addition (A7; 22\%) besides A4 (75\%), the sole product of $\mathrm{ArMgBr}$ addition. We thought that the bulky solubilizing $\mathrm{R}$ groups in A1 could steer the selectivity towards 1,4-addition due to steric hindrance. For this reason, we synthesized anthanthrone A8 equipped with sterically non-demanding (4-octylphenyl)ethynyl substituents. The reaction of $\mathbf{A 8}$ with PhCCLi proceeded as before in a 1,2-fashion (A9; 86\%), but $\mathrm{ArMgBr}$ perplexed us again —we isolated exclusively the product of a double 1,4-addition A10 (69\%, Scheme 2 and Table 1).

\subsection{General considerations}

According to the equation of Klopman and Salem, ${ }^{24}$ the reactivity of electrophiles and nucleophiles is governed by three terms: Coulombic interaction, orbital overlap and Pauli repulsion. When applied to $\alpha, \beta$-unsaturated carbonyl systems, the Coulombic term is in favor of 1,2-additions, while orbital overlap promotes 1,4-additions. For acene quinones, exclusively 1,2-additions with nucleophiles PhCCLi and ArMgBr are documented in the literature. ${ }^{12,17,18}$ In contrast, phenalenone, a compound of similar size but different conjugation topology, undergoes solely 1,4additions $^{23}$ (Figure 1). Clearly, the topological equivalence of triangulenedione and phenalenone leads to the same selectivity. On the other hand, the case of anthanthrone reveals that the rules, which govern selectivity, go beyond topology. Necessarily, the size of the nanographene core must be in play as well.

Indeed, we found three cases of extended Kekulé nanographene diones, where solely the products of 1,4-addition with ArMgBr were isolated. ${ }^{25}$ This unexpected selectivity, which has never been fully rationalized, might be the reason for the scarcity of the use of this method in the nanographene synthesis (see sections below). In what follows, we analyze the contribution of each term of the Klopman-Salem equation and provide the basis for predicting the selectivity of nucleophilic additions to zigzag nanographene ketones. 


\subsection{Theoretical insight}

We performed DFT calculations to determine charges ( $q$, Hirshfeld) and LUMO coefficients $(c)$ at the relevant positions of triangulenedione and anthanthrone. The former provides an estimate of the Coulombic term, while the latter the orbital overlap term (Figure 2). The carbonyl carbon atoms in both compounds bear the largest and similar positive charge and their coefficients in LUMO are smaller than those at the 1,4-positions. Compared to phenalenone and anthraquinone (Figure S2), the core extension leads to an increase of the $c 1,4 / c_{1,2}$ ratio, while the charges remain qualitatively the same. The same trend is observed upon longitudinal extension in acene quinones (Figure S3). Based on the documented regioselectivity of phenalenone and anthraquinone, it may appear that it is dominated by orbital overlap. This observation holds true also for non-Kekulé triangulenedione but in the case of anthanthrone the situation is more complex.

The charges and the change of the $c_{1,4} / c_{1,2}$ ratio with the size of the $\pi$-system do not allow to make clear-cut predictions in every case. For this reason, we calculated the Fukui functions (f), which express the change in electron density upon changing the number of electrons in the system. In our case, it is the change in electron density of the electrophile (ketone) upon addition of the nucleophilic electron pair. The Fukui function thus combines the Coulombic and the orbital overlap terms of the Klopman-Salem equation into one reactivity descriptor. We decomposed the Fukui function to individual positions in the ketones, an approach that has been successfully applied by others $^{26}$ to understand the observed experimental selectivity. Note, however, that the steric repulsion $(s)$ is not included in the Fukui function. The corresponding rate constants $(k)$ for the respective additions $(1,2$ or 1,4$)$ of a given nucleophile can be therefore thought of in terms of the following simplistic relations:

$$
\begin{aligned}
& k_{1,2} \sim f_{1,2} \times s_{1,2}-1 \\
& k_{1,4} \sim f_{1,4} \times s_{1,4}-1
\end{aligned}
$$




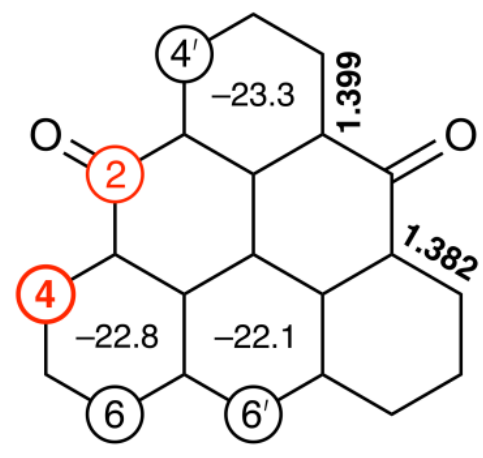

$\begin{array}{cccccc}\mathrm{A}_{N} & 1,2 & 1,4 & 1,4^{\prime} & 1,6 & 1,6^{\prime} \\ & & & & & \\ q & \mathbf{0 . 1 4 7} & 0.049 & 0.042 & 0.031 & 0.027 \\ |c| & 0.140 & \mathbf{0 . 2 9 7} & 0.022 & 0.304 & 0.422 \\ f & 0.028 & 0.054 & 0.023 & 0.048 & 0.060\end{array}$

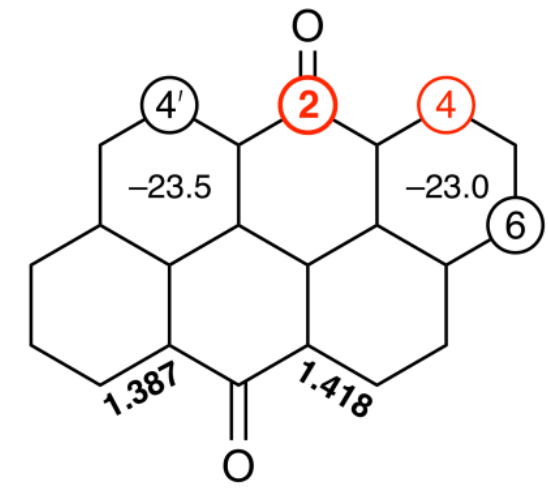

$1,2 \quad 1,4 \quad 1,4^{\prime} \quad 1,6$

$\begin{array}{llll}0.150 & 0.043 & 0.024 & 0.030\end{array}$

$\begin{array}{llll}0.194 & \mathbf{0 . 2 1 7} & 0.010 & 0.239\end{array}$

$\begin{array}{llll}0.044 & 0.041 & 0.020 & 0.045\end{array}$

Figure 2. Hirshfeld charges $(q)$, orbital coefficients in LUMO (c), and Fukui functions $(f)$ for triangulenedione and anthanthrone calculated by DFT (gray table); the highest out of two values for 1,2- and 1,4-additions is shown in bold. The NICS(1) values (ppm, ring centers) and C-C bond lengths $(\AA$, bold $)$ are shown in the structures.

\subsection{Analysis of triangulenedione}

The calculated charges $(q)$ reflect the expected trend (Figure 2, left). They decrease monotonically with increasing distance from the carbonyl center (2-4-6-6'). Non-intuitively, the orbital coefficients $(c)$ follow exactly the opposite trend, with the largest contribution to LUMO at position $6^{\prime}$. The Fukui function $(f)$, however, breaks the monotonous nature of these trends, with the highest values at positions $4(0.054)$ and $6^{\prime}(0.060)$. One would then assume that the nucleophilic addition to $\mathbf{T} 1$ takes place preferably at position $6^{\prime}$ in contrast to the experimental finding-exclusive attack at position 4 (Scheme 1, Table 1). This result suggests that metal coordination to carbonyl's oxygen atom is required for the reaction to take place. We confirmed this hypothesis by performing the addition to $\mathbf{T 1}$ with ArLi with and without DMPU as a chelating agent for $\mathrm{Li}$ ions. While ArLi forms the expected product $\mathbf{T} 2$ in $44 \%$ yield, we observed no reaction in the presence of DMPU 27 (Table 1). The same result was obtained with Kekulé anthanthrones A1 and A8 (Table 1). We can thus conclude that conjugate additions on nanographene diones require a directing group. In 
triangulenedione, 1,2- and two conjugate 1,4- and 1,4'-additions are therefore feasible and the Fukui function correctly predicts the experimental outcome. Indeed, a 1,4'-addition would disrupt one of the two Clar's sextets, unlike 1,4-addition (see Scheme 1). This result is further corroborated by the calculated $\mathrm{C}-\mathrm{C}$ bond lengths and NICS values (Figure 2).

\subsection{Analysis of anthanthrone}

As in triangulenedione, the Coulombic interaction clearly favors 1,2- over 1,4-addition, but the orbital overlap prefers 1,4- over 1,2-addition only moderately (Figure 2). As a result, the Fukui function at positions $2(0.044)$ and $4(0.041)$ have comparable values, with a slightly higher value at position 2. Accordingly, reaction of one equivalent of $\mathrm{ArMgBr}$ with $\mathbf{A 1}$ displays both selectivities, but with a preference for 1 ,4-addition $\left(k_{1,4} / k_{1,2} \sim 3: 2\right.$, Scheme 2 , Table 1$)$. Reactions with PhCCLi, however, differ and give exclusively the products of 1,2-addition. This observation is not so surprising because the Fukui function does not include Pauli repulsive interactions and the sizes of these two nucleophiles significantly differ. Bürgi and Dunitz showed that nucleophiles attack carbonyl centers under an ideal angle of $107^{\circ} .{ }^{28}$ This means that the nucleophile can experience a steric clash with the $\pi$-electron cloud in extended nanographene ketones (Figure 3 ).

\section{top view}

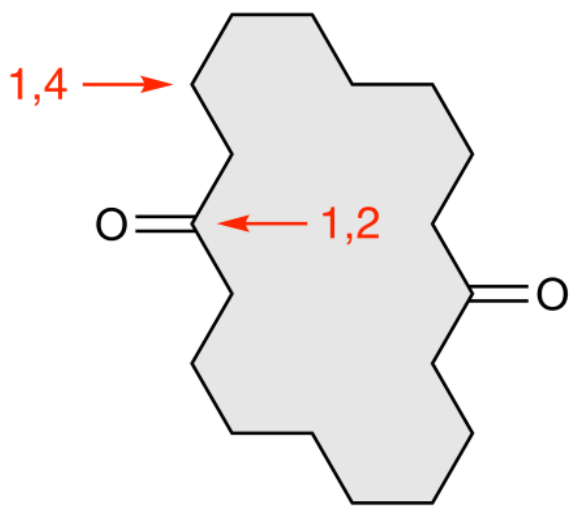

side view

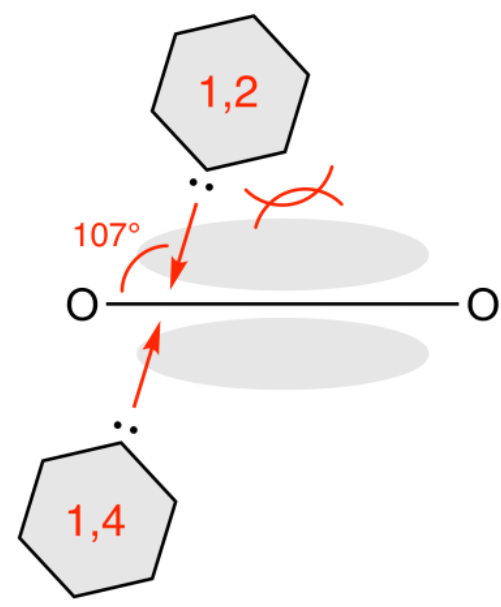

Figure 3. Illustration of the effect of the Pauli repulsion on the nucleophilic 1,2- and 1,4-addition to anthanthrone. 
On the other hand, the nucleophile in a conjugate addition attacks the reactive center at the edge from the outside of the nanographene core. Consequently, 1,2-additions suffer more from Pauli repulsion and in the case of bulkier nucleophiles, such as aryls, the Pauli term can outweigh the other two terms in the Klopman-Salem equation, steering the selectivity towards conjugate addition. The excess of $\mathrm{ArMgBr}$ gives a single product of a mixed 1,2-/1,4-addition in a very good yield (83\%), which indicates that each of the two intermediates A12 and A13 (Figure 4) formed after the first $\mathrm{ArMgBr}$ addition undergoes a selective complementary second addition (Scheme 2). That means that $\mathbf{A 1 2}$ undergoes a 1,4-addition and $\mathbf{A 1 3}$ a 1,2-addition. While the latter clearly follows the prediction of the Fukui functions (Figure 4, right), the former is a borderline case, where the sterics must overweigh the minor electronic preference for 1,2- in favor of 1,4-addition. If the steric repulsion has such a strong effect, then one expects that increasing the steric bulk of the nucleophile will push the selectivity in favor of 1,4-addition even more in each of the two steps. In an extreme case, even a product of a double 1,4-addition could form. To confirm this hypothesis, we performed the reaction of $\mathbf{A 1}$ with an excess of mesityl magnesium bromide (MesMgBr, Table 1) and isolated exclusively the product of a double 1,4-addition A11 (91\%). The same change of selectivity upon increasing steric bulk of the nucleophile was observed for benzophenalenone. ${ }^{29}$ The steric argument holds true also for the case of 1,2-addition of thienyl lithium to anthanthrone, ${ }^{19 \mathrm{a}}$ as thienyl is less bulky than 3,5-di-tert-butylphenyl.

The reaction of $\mathbf{A 1}$ with an excess of ArLi affords two products: mixed A4 (75\%, Table 1) and the product of a double 1,4-addition A7 (22\%). The formation of A7 is unexpected considering that the size of nucleophile (Ar) did not change compared to $\mathrm{ArMgBr}$ and organolithiums are known to favor 1,2-additions. ${ }^{30}$ Because in ArLi the $\mathrm{C}-\mathrm{M}$ bond is more polarized than in $\mathrm{ArMgBr}$, $\mathrm{ArLi}$ is more basic and reactive, which might give an impression that it is also harder. In our reactions, however, $\mathrm{ArLi}$ appears to be softer than $\mathrm{ArMgBr}$, which is in agreement with calculated average local ionization energies (PhLi: $6.0 \mathrm{eV}, \mathrm{PhMgBr}: 8.2 \mathrm{eV}$ ). ${ }^{31}$ 
1,2-addition<smiles></smiles>

A12: $\mathrm{M}=\mathrm{MgBr}$ 1,4-addition

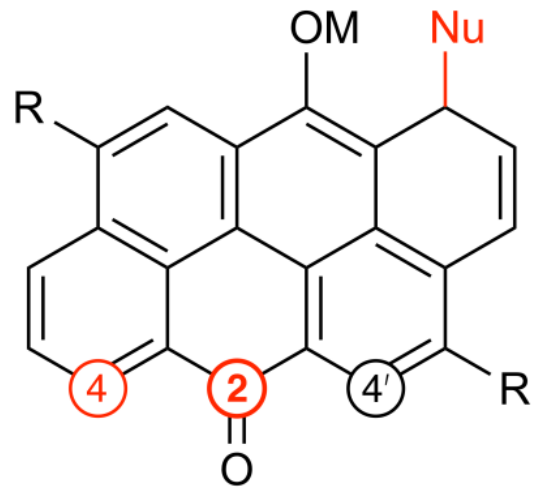

A13: $\mathrm{M}=\mathrm{MgBr}$

$\begin{array}{cccccccc}A_{N} & 1,2 & 1,4 & 1,4^{\prime} & & 1,2 & 1,4 & 1,4^{\prime} \\ q & \mathbf{0 . 1 4 7} & 0.041 & 0.019 & & \mathbf{0 . 1 3 4} & -0.006 & -0.030 \\ |c| & 0.239 & \mathbf{0 . 2 9 7} & 0.012 & & 0.303 & \mathbf{0 . 3 0 5} & 0.249 \\ f & 0.054 & 0.053 & 0.015 & & 0.065 & 0.050 & 0.044\end{array}$

Figure 4. Hirshfeld charges ( $q$ ), orbital coefficients in LUMO (c), and Fukui functions $(f)$ for A12 and $\mathbf{A 1 3}(\mathrm{R}=\mathrm{Ar}=3,5$-di-tert-butylphenyl) calculated by DFT (gray table); the highest out of two values for 1,2- and 1,4-additions is shown in bold; calculations were carried out for $\mathrm{R}=\mathrm{Nu}=\mathrm{M}=$ $\mathrm{H}(\mathbf{A 1 2})$ and $\mathrm{R}=\mathrm{Nu}=\mathrm{H}, \mathrm{OM}=\mathrm{O}^{-}(\mathbf{A 1 3})$.

To exclude any steric effect of the R substituent on 1,2-additions, we tested the same reactions on A8 equipped with sterically non-demanding substituents ( $\mathrm{R}=$ (4-octylphenyl)ethynyl), Scheme 2, Table 1). Both $\mathrm{ArMgBr}$ and ArLi gave the same unexpected result, a single product of a double 1,4-addition. This rules out the steric effect of the $\mathrm{R}$ groups and points at an enhanced electronic effect. Indeed, the ethynyl groups perturb the frontier molecular orbitals and affect the Fukui functions. While in $\mathbf{A 1}$ and $\mathbf{A 1 3}$, the Fukui functions favor 1,2-addition, this preference diminishes with ethynyl side groups (Figure S5). Even though the change is subtle, it has a profound consequence on selectivity. 


\subsection{Rules of thumb}

Our observations can be summarized into the following basic set of rules for nucleophilic additions to zigzag nanographene ketones:

1. A directing group is required.

2. Conjugation topology affects the electronic preference of 1,2- versus 1,4-selectivity, expressed by $f_{1,4} / f_{1,2}$ ratio (Table 2 , vertical).

3. Increasing the extent of $\pi$-electron cloud and size of the nucleophile steers the selectivity towards 1,4-addition (Table 2, row 2).

4. Steric bulk imposed by additional substituents at or close to the reactive position or nonplanar geometry may alter the expected selectivity (see Applications section).

Table 2. Rules of thumb

\begin{tabular}{ccc}
\hline Nu $(\rightarrow)$ & non-bulky & bulky \\
dione $(\downarrow)$ & $($ e.g., PhCCLi) & (e.g., ArMgBr) \\
\hline$f_{1,4} / f_{1,2}>1^{a}$ & $\mathbf{1 , 4}$ & $\mathbf{1 , 4}$ \\
$f_{1,4} / f_{1,2} \sim 1^{b}$ & (phenalenone) & (triangulenedione) \\
$f_{1,4} / f_{1,2}<1^{c}$ & (anthanthrone) & $\mathbf{1 , 2}$ and $\mathbf{1 , 4}$ \\
(anthanthrone)
\end{tabular}

${ }^{a}$ Typical for non-Kekulé conjugation topology (possible also for Kekulé, see Applications section). ${ }^{b}$ Possible for both non-Kekulé and Kekulé conjugation topology. ${ }^{c}$ Typical for Kekulé conjugation topology.

\subsection{Applications}

In the chemical literature, nucleophilic addition is uncommon as a method to functionalize nanographene ketones. Our results show that the reason for this might be that a mixture of products is often formed, in particular if an excess of nucleophile is used. Indeed, we found three reports describing this issue. ${ }^{25 a, 32}$ Although in some cases, products can be formed selectively and in good yields, understanding the principles that govern these reactions is crucial to control the selectivity. Below, we test the predictive power of our analysis on three examples from the literature. 
In two reported reactions of extended Kekulé nanographene diones with $\mathrm{ArMgBr}, 1,4$-addition products were obtained in good yields (Scheme 3). The "butterfly" system from Zhang et al. $(2010)^{25 c}$ is analogous to anthanthrone, while the second one $e^{25 b}$ to anthraquinone. In the former case, Fukui functions show a preference for 1,2-addition, like in anthanthrone (rule no. 2). We would, however, expect that the steric clash between nucleophile and the "butterfly arms" of the nanographene dione significantly increases the magnitude of the steric repulsion compared to anthanthrone, which should outweigh the electronic preference (rule no. 4). Indeed, the unexpected Michael addition is the experimental outcome. ${ }^{25 \mathrm{c}}$

Scheme 3. Application to examples from literature

"butterfly" dione by Zhang et al. (2010)

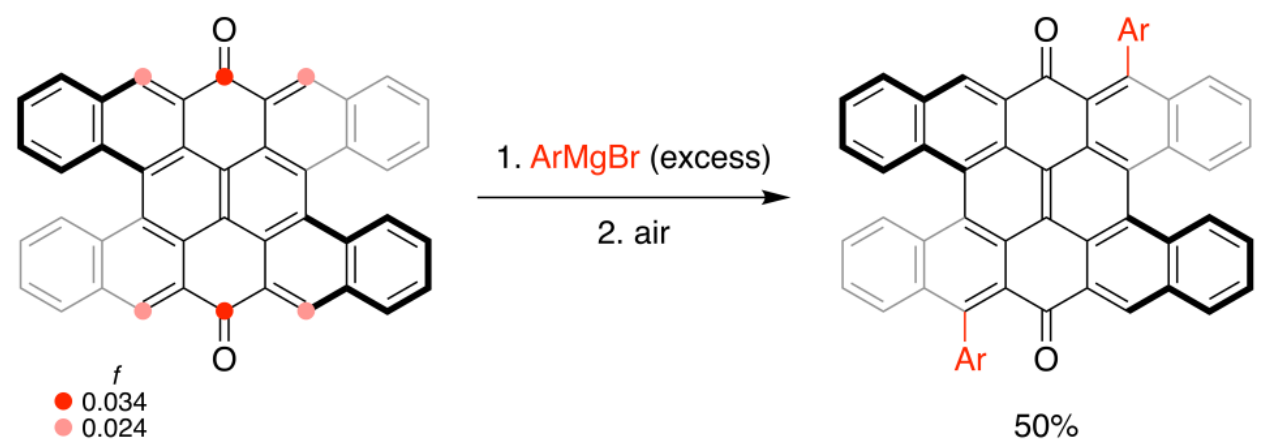

extended hexacenedione by Li et al. (2011) ${ }^{a}$

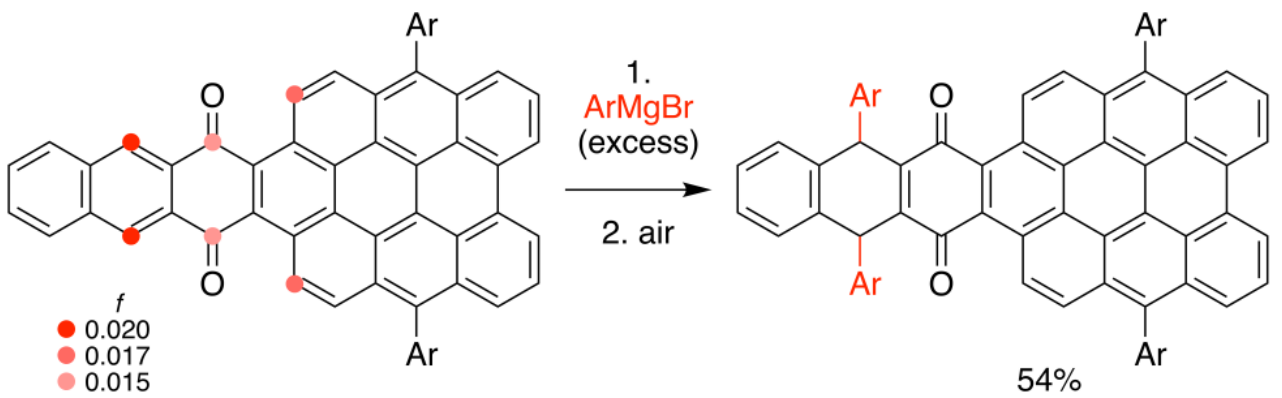

${ }^{a}$ Note that only a partial oxidation in air of the intermediate hydroquinone obtained after the workup takes place. Further dehydrogenation did not occur even upon heating the product in the presence of $p$-chloranil at reflux.

The Fukui functions calculated for the unsymmetrically extended hexacenequinone system from Li et al. (2011) $)^{25 b}$ favor 1,4-addition, unlike in anthraquinone or anthanthrone. In this scenario, 1,4-addition should be the unequivocal result, which is the case (rule no. 2). 
An intriguing observation was made during the synthesis of persistent [7]uthrene, another nonKekulé triplet diradical (Li et al., 2014). ${ }^{33}$ The first attempted synthetic route relied on a double 1,2-addition to [7] uthrenedione (U1, Scheme 4). The use of an excess of MesMgBr led to a complex reaction mixture and no desired product could be detected. It led the authors to re-design the synthesis and to install the substituents by a nucleophilic addition to a dialdehyde prior to the nanographene core formation by the Friedel-Crafts alkylation. Once we calculated the Fukui functions for $\mathbf{U} 1$ (Figure S6), this system caught our attention. Analogously to triangulenedione, no 1,2- but 1,4-addition is predicted (rule no. 2). We therefore re-synthesized $\mathbf{U} \mathbf{1}$ and tested the selectivity of additions with ArMgBr. Stepwise addition/oxidation provided 1,4-addition products U2 $(22 \%$, Scheme 4$)$ and $\mathbf{U} 3$ (26\%). The reactions with one equivalent of ArMgBr were selective but slow and the unreacted starting material was recovered. Interestingly, the addition of a large excess of ArMgBr to $\mathbf{U} 3$ afforded $\mathbf{U} 4$ (55\%), the product of 1,2-addition, despite a lower $f$ value $\left(f_{1,4} / f_{1,2} \sim 1.2\right)$. We attribute this change in the selectivity to a steric hindrance imposed by the substituent present in position 4 that must be larger than that of the $\pi$-electron cloud (rule no. 4), size of which is intermediate between those of acene quinones and anthanthrone.

Scheme 4. Nucleophilic additions to [7] uthrenedione

explanation of observation by Li et al. (2014)<smiles>Cc1cc(C)c(-c2c(C)ccc3cccc(C)c23)cc1C(=O)Oc1cccc(C(=O)O)c1</smiles>

U1

- 0.037

0.031<smiles>Cc1ccc2ccc(Br)c3c2c1-c1cc2c(cc1C3=O)C(O)(Br)c1c(Br)ccc3ccc(Br)c-2c13</smiles>

U4, $55 \%$
1. $\mathrm{ArMgBr}$ (1 equiv)<smiles>Cc1ccc2cccc3c(=O)c4cc5c(=O)c6c(Br)ccc7ccc(Br)c(c5cc4c1c23)c76</smiles>

U2, $22 \%$

ArMgBr (40 equiv)

1. $\mathrm{ArMgBr}$ (1 equiv)

2. $p$-chloranil<smiles>Cc1ccc2ccc(Br)c3c(=O)c4cc5c(cc4c1c23)c(=O)c1c(Br)ccc2ccc(C)c5c21</smiles>

U3, $26 \%$ 
To test if triangulenedione gives a similar result as [7]uthrenedione, we performed the second and the third addition with $\mathrm{ArMgBr}$ to $\mathbf{T} 2$ (Scheme 5). The second addition/oxidation proceeded as expected and the Ar group was installed at the second, unsubstituted position 4, affording $\mathbf{T} 3$ in an excellent yield (98\%). Interestingly, the third addition also gave a product of 1,4-addition T5 (53\%), even though the addition occurred at a position already bearing one Ar substituent (Table 2, row 1).

Scheme 5. More nucleophilic additions ${ }^{a}$ to $\mathbf{T 2}$

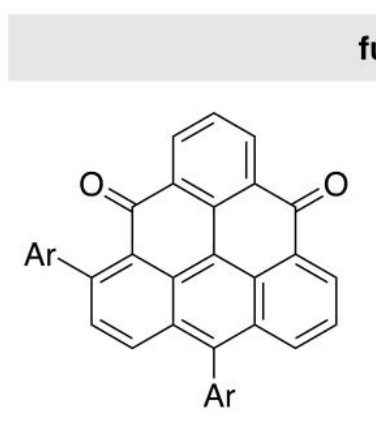

T2

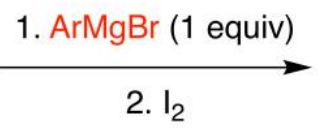

2. $\mathrm{I}_{2}$ 1. $\mathrm{ArMgBr}$ (0.95 equiv to T5) (40 equiv to $\mathrm{T6}$ )<smiles></smiles>

T6, 37\%

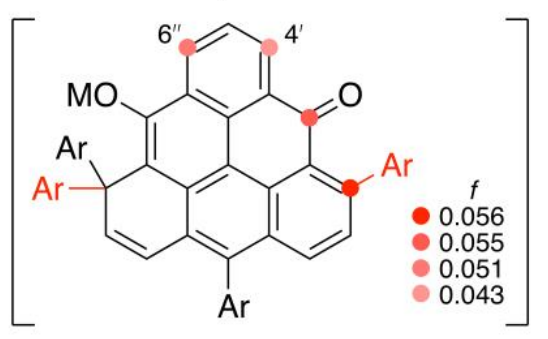

2. $\mathrm{H}_{2} \mathrm{O} \longrightarrow \begin{array}{r}\text { T4, } M=M g B r \\ \text { T5, } M=H, 53 \%\end{array}$

${ }^{a}$ Calculations of the Fukui functions $(f)$ were carried out for triangulenedione (T3) and $\mathrm{OM}=\mathrm{O}^{-}$ (T4).

Compared to [7]uthrenedione, the steric hindrance of the Ar substituent does not overweigh the more pronounced electronic preference of triangulenedione for 1,4-addition $\left(f_{1,4} / f_{1,2} \sim 2\right.$, rule no. 2) combined with the steric hindrance imposed by the larger $\pi$-electron cloud disfavoring 1,2addition (rule no. 3). When an excess of $\mathrm{ArMgBr}$ was reacted with $\mathbf{T 3}$, a product of 1,4- and subsequent 1,6-addition T6 was isolated (37\%). To the best of our knowledge, this is the first 
example of 1,6-addition on a nanographene dione. It can be rationalized by the fact that (1) the Fukui function at position 6" of intermediate T4 (Figure S7) formed upon 1,4-addition is comparable to those at positions 2 and 4, hindered by Ar group, and larger than that in position 4' and (2) metal coordination is possible with the oxygen atom of the oxy group, which acts as the directing group (rule no. 1). This result demonstrates the versatility of this method by judicious placement of a suitable directing group.

\section{Conclusion}

We explored the regioselectivity of nucleophilic additions of carbon-centered nucleophiles $\mathrm{ArMgBr}$ and PhCCLi to zigzag nanographene ketones, namely, non-Kekulé triangulene-4,8-dione and Kekulé anthanthrone. We observed a number of unexpected results and unequivocally rationalized them as an interplay of electronic effects, expressed by Fukui functions, and sterics related to the size of nanographene and nucleophile. We compiled our findings into a basic set of rules that govern these reactions (Table 2), the use of which is rare in the chemical literature. These principles helped us explain the few known examples with unanticipated selectivity and can be potentially extended to other types of reactions, such as transition-metal-catalyzed $\mathrm{C}-\mathrm{H}$ activation. The synthesis of new nanographenes aids and abets understanding of physical phenomena in graphene-based materials, important for the future development of organic electronics. Functionalization of their periphery is crucial to control their electronic structure, solubility, stability, and self-assembly behavior. In this respect, the selectivity rules of nucleophilic additions to zigzag nanographene ketones developed in this work do not only allow to install substituents at a late stage of the synthesis, but make it possible to address positions, which would not be accessible otherwise. In addition, this method will facilitate synthesis and exploration of new functionalized nanographenes as a large number of nanographene ketones have been described since the pioneering work of Eric Clar.

\section{Experimental section}

Experimental procedures and characterization data for all new compounds described in this work are compiled in the Supporting Information (sections S2 and S3). Compound T1 was prepared from 12-hydroxydibenzo[cd,mn]pyrene-4,8-dione described previously ${ }^{34}$ in two steps. All chemicals and solvents were purchased from commercial sources and were used without further purification unless stated otherwise. The reactions and experiments that are sensitive to dioxygen 
were performed using Schlenk techniques and nitrogen or argon-saturated solvents. The NMR experiments were performed on NMR spectrometers operating at 400, 500, or $600 \mathrm{MHz}$ proton frequencies. Standard pulse sequences were used. Chemical shifts $(\delta)$ are reported in parts per million (ppm) relative to the solvent residual peak $\left({ }^{1} \mathrm{H}\right.$ and ${ }^{13} \mathrm{C} \mathrm{NMR}$, respectively): $\mathrm{CDCl}_{3}(\delta=$ 7.26 and $\left.77.16 \mathrm{ppm}^{35}\right), \mathrm{CD}_{2} \mathrm{Cl}_{2}\left(\delta=5.32\right.$ and $\left.53.84 \mathrm{ppm}^{35}\right)$, and $\mathrm{C}_{2} \mathrm{D}_{2} \mathrm{Cl}_{4}(\delta=6.00$ and 73.78 $\mathrm{ppm}^{36}$ ). High-resolution mass spectra (HRMS) were measured as HR-ESI-MS or HR-APCI-MS.

\subsection{Preparation of organometallic reagents}

Grignard reagents were titrated according to the procedure of Knochel ${ }^{37}$ and organolithium reagents according to the procedure of Chong. ${ }^{38}$ A solution of (3,5-di-tert-butylphenyl)magnesium bromide in dry THF was prepared according to the following procedure: Magnesium powder was washed with aq. $\mathrm{HCl}(1 \mathrm{M})$, filtered through the filter frit, washed with $\mathrm{H}_{2} \mathrm{O}, \mathrm{MeOH}$, and $\mathrm{Et}_{2} \mathrm{O}$, and dried under high vacuum overnight. Freshly pre-activated magnesium powder was placed into a Schlenk flask and annealed with heat-gun $\left(200{ }^{\circ} \mathrm{C}\right.$, vacuum, $\left.10 \mathrm{~min}\right)$ before a tiny crystal of iodine $(\sim 1 \mathrm{mg})$ was added under a nitrogen atmosphere. This mixture was annealed again with a heat-gun $\left(200^{\circ} \mathrm{C}, \mathrm{N}_{2}, 10 \mathrm{~min}\right)$ until all iodine vaporized. Excessive iodine vapors were removed under high vacuum and the flask was allowed to cool to room temperature $\left(\mathrm{N}_{2}\right)$. Then, a solution of 1-bromo-3,5-di-tert-butylbenzene in dry THF $(c \sim 100 \mathrm{mg} / \mathrm{mL})$ was added, the Schlenk flask was closed, and the reaction mixture was heated at $55^{\circ} \mathrm{C}$ until the solution became cloudy (usually for $\sim 1 \mathrm{~h}$ ). The reaction mixture was allowed to cool to room temperature and titrated before use.

\subsection{DFT Calculations}

All calculations were performed with Gaussian 09 (ver. D01) package of electronic structure programs. ${ }^{39}$ The gas phase geometries were optimized with B3LYP functional and 6-31G(d) basis set. The frequency analysis of the minimum energy geometries confirmed that they represented the potential energy surface minima. The isotropic NMR chemical shielding tensors and nucleusindependent chemical shifts ${ }^{40}\left(\mathrm{NICS}_{z z}\right.$ at $\left.1 \AA \AA\right)$ were calculated at the B3LYP/6-31+G(d)/GIAO level of theory and tetramethylsilane was used as the reference. Hirshfeld charges in individual positions were computed at the B3LYP/6-31G(d) level of theory. The charges with the hydrogen atoms summed into carbon atoms are reported, while "naked" charges have been used in the calculation of the condensed Fukui functions. The reported condensed Fukui functions are the difference between the Hirshfeld charge of the neutral molecule and its corresponding radical 
anion, both calculated in the geometry of the neutral molecule. This approach has been successfully used before to predict reactivity of organic molecules. ${ }^{26}$ Orbital coefficients were obtained from B3LYP Kohn-Sham wavefunctions with minimal STO-3G basis set. The coefficients were comparable to those obtained when a larger 6-31G(d) basis set was used in several test cases.

\section{Acknowledgement}

This project received funding from the European Research Council (ERC) under the European Union's Horizon 2020 research and innovation programme (Grant Agreement No. 716139), the Swiss National Science Foundation (SNSF, T.Š./PZ00P2_174175, M.J./PZ00P2_148043, PP00P2_170534), and Experientia Foundation (T.Š.). We would like to thank Prof. Marcel Mayor (University of Basel) for his generous support of our research.

\section{References}

${ }^{1}$ a) Yamaguchi, J.; Hayashi, H.; Jippo, H.; Shiotari, A.; Ohtomo, M.; Sakakura, M.; Hieda, N.; Aratani, N.; Ohfuchi, M.; Sugimoto, Y.; Yamada, H.; Sato, S., Commun. Mater. 2020, 1, 36; b) Son, Y.-W.; Cohen, M. L., Louie, S. G., Phys. Rev. Lett. 2006, 97, 216803.

2 a) Yazyev, O. V., Rep. Prog. Phys. 2010, 73, 056501; b) Jung, J.; Pereg-Barnea, T.; MacDonald, A. H., Phys. Rev. Lett. 2009, 102, 227205; c) Kim, W. Y.; Kim, K. S., Nat. Nanotech. 2008, 3, 408412.

${ }^{3}$ a) Grzybowski, M.; Sadowski, B.; Butenschön, H.; Gryko, D. T., Angew. Chem. Int. Ed. 2020, 59, 2998-3027; b) Senese, A. D.; Chalifoux. W. A., Molecules 2019, 24, 118; c) Narita, A.; Wang, X.-Y.; Feng, X.; Müllen, K., Chem. Soc. Rev. 2015, 44, 6616-6643.

${ }^{4}$ a) Wang, X.-Y.; Yao, X.; Müllen, K., Sci. China Chem. 2019, 62, 1099-1144; b) Wu, J.; Pisula, W.; Müllen, K., Chem. Rev. 2007, 107, 718-747.

5 a) Inoue, J.; Fukui, K.; Kubo, T.; Nakazawa, S.; Sato, K.; Shiomi, D.; Morita, Y.; Yamamoto, K.; Takui, T.; Nakasuji, K., J. Am. Chem. Soc. 2001, 123, 12702-12703; b) Goto, K.; Kubo, T.; Yamamoto, K.; Nakasuji, K.; Sato, K.; Shiomi, D.; Takui, T.; Kubota, M.; Kobayashi, T.; Yakusi, K.; Ouyang, J. Y., J. Am. Chem. Soc. 1999, 121, 1619-1620.

${ }^{6}$ Simpson, C. D.; Brand, J. D.; Berresheim, A.J.; Przybilla, L.; Räder, H. J.; Müllen, K., Chem. Eur. J. 2002, 8, 1424-1429. 
7 a) Anthony, J. E., Angew. Chem. Int. Ed. 2008, 47, 452-483; b) Anthony, J. E., Chem. Rev. 2006, 106, 5028-5048.

8 a) Mei, J.; Diao, Y.; Appleton, A. L.; Fang, L.; Bao, Z., J. Am. Chem. Soc. 2013, 135, 67246746; b) Watanabe, M.; Chang, Y. J.; Liu, S.-W.; Chao, T.-H.; Goto, K.; Islam, Md. M.; Yuan, C.H.; Tao, Y.-T.; Shinmyozu, T.; Chow, T. J., Nat. Chem. 2012, 4, 574-578.

9 a) Rieger, R.; Müllen, K., J. Phys. Org. Chem. 2010, 23, 315-325; b) Koch, K.-H.; Müllen, K., Chem. Ber. 1991, 124, 2091-2100.

${ }^{10}$ a) Purushothaman, B.; Bruzek, M.; Parkin, S. R.; Miller, A.-F.; Anthony, J. E., Angew. Chem. Int. Ed. 2011, 50, 7013-7017; b) Payne, M. M.; Parkin, S. R.; Anthony, J. E., J. Am. Chem. Soc. 2005, 127, 8028-8029.

11 a) Kaur, I.; Jazdzyk, M.; Stein, N. N.; Prusevich, P.; Miller, G. P., J. Am. Chem. Soc. 2010, 132, 1261-1263; b) Qu, H.; Chi, C. A., Org. Lett. 2010, 12, 3360-3363.

12 Anthony, J. E.; Brooks, J. S.; Eaton, D. L.; Parkin, S. R., J. Am. Chem. Soc. 2001, 123, 94829483.

13 a) Ito, H.; Segawa, Y.; Murakami, K.; Itami, K., J. Am. Chem. Soc. 2019, 141, 3-10; b) Mathew, B. P.; Kuram, M. R., Inorganica Chim. Acta 2019, 490, 112-129; c) Ito, H.; Ozaki, K.; Itami, K., Angew. Chem. Int. Ed. 2017, 56, 11144-11164.

14 a) Gu, Y.; Tullimilli, Y. G.; Feng, J.; Phan, H.; Zeng, W.; Wu, J., Chem. Commun. 2019, 55, 5567-5570; b) Zeng, W.; Gopalakrishna, T. Y.; Phan, H.; Tanaka, T.; Herng, T. S.; Ding, J.; Osuka, A.; Wu, J., J. Am. Chem. Soc. 2018, 140, 14054-14058; c) Gu, Y.; Wu, X.; Gopalakrishna, T. Y.; Phan, H.; Wu, J., Angew. Chem. Int. Ed. 2018, 57, 6541-6545.

15 a) Clar, E.; Schoental, R. Polycyclic Hydrocarbons: Volume 1; Springer Berlin / Heidelberg: Berlin, Heidelberg, 2013; b) Clar, E. Polycyclic Hydrocarbons: Volume 2; Springer Berlin / Heidelberg: Berlin, Heidelberg, 2013.

${ }^{16}$ Examples with lithium acetylide: a) Lehnherr, D.; Gao, J.; Hegmann, F. A.; Tykwinski, R. R., Org. Lett. 2008, 10, 4779-4782; b) Taylor, M. S.; Swager, T. M., Org. Lett. 2007, 9, 3695-3697; c) Lydon, D. P.; Porrès, L.; Beeby, A.; Marder, T. B.; Low, P. J., New J. Chem. 2005, 29, 972-976. Examples with (hetero)aryl lithium: d) Voll, C.-C. A.; Swager, T. M., J. Am. Chem. Soc. 2018, 140, 17962-17967; e) Gessner, V. H.; Tilley, T. D., Org. Lett. 2011, 13, 1154-1157; f) Wang, J.; Liu, K.; Liu, Y.-Y.; Song, C.-L.; Shi, Z.-F.; Peng, J.-B.; Zhang, H.-L.; Cao, X.-P., Org. Lett. 2009, 11, 2563-2566; g) Kaur, I.; Jia, W.; Kopreski, R. P.; Selvarasah, S.; Dokmeci, M. R.; Pramanik, C.; 
McGruer, N. E.; Miller, G. P., J. Am. Chem. Soc. 2008, 130, 16274-16286. Examples with (hetero)aryl magnesium bromide: h) Chalke, R. M.; Patil, V. R., Polymer 2017, 123, 355-365; i) Kim, D.-S.; Jung, J. E.; Baek, N. S.; Kim, T.-D., Org. Electron. 2015, 17, 319-324.

17 a) Purushothaman, B.; Parkin, S. R.; Anthony, J. E., Org. Lett. 2010, 12, 2060-2063.

18 a) Giguère, J.-B.; Boismenu-Lavoie, J.; Morin, J.-F., J. Org. Chem. 2014, 79, 2404-2418; b) Matsuno, T.; Kamata, S.; Hitosugi, S.; Isobe, H., Chem. Sci. 2013, 4, 3179-3183; c) Giguère, J.B.; Verolet, Q.; Morin, J.-F., Chem. Eur. J. 2013, 19, 372-381; d) Zhang, L.; Walker, B.; Liu, F.; Colella, N. S.; Mannsfeld, S. C. B.; Watkins, J. J.; Nguyen, T.-Q.; Briseno, A. L., J Mater Chem 2012, 22, 4266-4268.

${ }^{19}$ Shah, B. K.; Neckers, D. C.; Shi, J.; Forsythe, E. W.; Morton, D., J. Phys. Chem. A 2005, 109, $7677-7681$.

${ }^{20}$ Phenalenyl has an odd number of carbon atoms and is not, strictly speaking, a non-Kekulé system. Nevertheless, it is generally considered to have non-Kekulé topology because of similar distribution of unpaired electrons compared to non-Kekulé systems such as triangulene. For IUPAC definition of non-Kekulé systems, see: Minkin, V. I., Pure Appl. Chem. 1999, 71, 1919.

21 Pavliček, N.; Mistry, A.; Majzik, Z.; Moll, N.; Meyer, G.; Fox, D. J.; Gross, L., Nat. Nanotechnol. 2017, 12, 308-311.

${ }^{22}$ Clar, E.; Stewart, D. G., J. Am. Chem. Soc. 1953, 75, 2667-2672.

${ }^{23}$ a) Wehrmann, C. M.; Charlton, R. T.; Chen, M. S., J. Am. Chem. Soc. 2019, 141, 3240-3248; b) Rosquete, L. I.; Cabrera-Serra, M. G.; Piñero, J. E.; Martín-Rodríguez, P.; Fernández-Pérez, L.; Luis, J. G.; McNaughton-Smith, G.; Abad-Grillo, T., Bioorg. Med. Chem. 2010, 18, 4530-4534; c) Newman, M. S.; Lee, L.-F., J. Org. Chem. 1975, 40, 2650-2652.

${ }^{24}$ a) Fleming, I. Ionic Reactions. Frontier Orbitals and Organic Chemical Reactions; John Wiley \& Sons: Chichester, 1976; pp 33-85; b) Klopman, G., J. Am. Chem. Soc. 1968, 90, 223-234; c) Salem, L., J. Am. Chem. Soc. 1968, 90, 543-552.

${ }^{25}$ a) Reus, C.; Lechner, M. P.; Schulze, M.; Lungerich, D.; Diner, C.; Gruber, M.; Stryker, J. M.; Hampel, F.; Jux, N.; Tykwinski, R. R., Chem. Eur. J. 2016, 22, 9097-9101; b) Li, J.; Jiao, C.; Huang, K.-W.; Wu, J., Chem. Eur. J. 2011, 17, 14672-14680; c) Zhang, X.; Li, J.; Qu, H.; Chi, C.; Wu, J., Org. Lett. 2010, 12, 3946-3949. 
${ }^{26}$ a) Wang, B.; Rong, C.; Chattaraj, P. K.; Liu, S., Theor. Chem. Acc. 2019, 138, 124; b) Liu, S.; Rong, C.; Lu, T., J. Phys. Chem. A 2014, 118, 3698-3704; c) Roy, R. K., J. Phys. Chem. A 2003, 107, 10428-10434.

${ }^{27}$ Chelation of Li ions by DMPU was shown to increase the reactivity of the nucleophile (which we did not observe in our case) and to alter the selectivity of the nucleophilic addition to cyclohexenone from 1,2 to 1,4: Mukhopadhyay, T.; Seebach, D., Helv. Chim. Acta 1982, 65, 385391.

${ }^{28}$ Bürgi, H. B.; Dunitz, J. D.; Lehn, J. M.; Wipff, G., Tetrahedron 1974, 30, 1563-1572.

${ }^{29}$ Debeaux, M.; Brandhorst, K.; Jones, P. G.; Hopf, H.; Grunenberg, J.; Kowalsky, W.; Johannes, H.-H., Beilstein J. Org. Chem. 2009, 5, 31.

${ }^{30}$ Amoah, E.; Dieter, R. K., J. Org. Chem. 2017, 82, 2870-2888 and references cited therein.

${ }^{31}$ Brown, J. J.; Cockroft, S. L., Chem. Sci. 2013, 4, 1772-1780.

32 a) Hu, P.; Lee, S.; Park, K. H.; Das, S.; Herng, T. S.; Gonçalves, T. P.; Huang, K.-W.; Ding, J.; Kim, D.; Wu, J., J. Org. Chem. 2016, 81, 2911-2919; b) Ye, Q.; Chang, J.; Huang, K.-W.; Dai, G.; Zhang, J.; Chen, Z.-K.; Wu, J.; Chi, C., Org. Lett. 2012, 14, 2786-2789.

${ }^{33}$ Li, Y.; Huang, K.-W.; Sun, Z.; Webster, R. D.; Zeng, Z.; Zeng, W.; Chi, C.; Furukawa, K.; Wu, J., Chem. Sci. 2014, 5, 1908-1914.

34 Ribar, P.; Šolomek, T.; Juríček, M., Org. Lett. 2019, 21, 7124-7128.

${ }^{35}$ Fulmer, G. R.; Miller, A. J. M.; Sherden, N. H.; Gottlieb, H. E.; Nudelman, A.; Stoltz, B. M.; Bercaw, J. E.; Goldberg, K. I., Organometallics 2010, 29, 2176-2179.

${ }^{36}$ Budavari, S.; O’Neil, M. J.; Smith, A.; Heckelman, P. E. The Merck Index, an encyclopedia of chemicals, drugs, and biologicals - eleventh edition; Merck Co., Inc.: Rahway, NJ, 1989.

${ }^{37}$ Krasovskiy, A.; Knochel, P., Synthesis 2006, 0890-0891.

${ }^{38}$ Burchat, A. F.; Chong, J. M.; Nielsen, N., J. Organomet. Chem. 1997, 542, 281-283.

39 Gaussian 09, Revision D.01, Frisch, M. J.; Trucks, G. W.; Schlegel, H. B.; Scuseria, G. E.; Robb, M. A.; Cheeseman, J. R.; Scalmani, G.; Barone, V.; Mennucci, B.; Petersson, G. A.; Nakatsuji, H.; Caricato, M.; Li, X.; Hratchian, H. P.; Izmaylov, A. F.; Bloino, J.; Zheng, G.; Sonnenberg, J. L.; Hada, M.; Ehara, M.; Toyota, K.; Fukuda, R.; Hasegawa, J.; Ishida, M.; Nakajima, T.; Honda, Y.; Kitao, O.; Nakai, H.; Vreven, T.; Montgomery, J. A. Jr.; Peralta, J. E.; Ogliaro, F.; Bearpark, M.; Heyd, J. J.; Brothers, E.; Kudin, K. N.; Staroverov, V. N.; Kobayashi, R.; Normand, J.; Raghavachari, K.; Rendell, A.; Burant, J. C.; Iyengar, S. S.; Tomasi, J. ; Cossi, 
M.; Rega, N.; Millam, J. M.; Klene, M.; Knox, J. E.; Cross, J. B.; Bakken, V.; Adamo, C.; Jaramillo, J.; Gomperts, R.; Stratmann, R. E.; Yazyev, O.; Austin, A. J.; Cammi, R.; Pomelli, C.; Ochterski, J. W.; Martin, R. L.; Morokuma, K.; Zakrzewski, V. G.; Voth, G. A.; Salvador, P.; Dannenberg, J. J.; Dapprich, S.; Daniels, A. D.; Farkas, Ö.; Foresman, J. B.; Ortiz, J. V.; Cioslowski, J.; Fox, D. J. Gaussian, Inc., Wallingford CT, 2009.

40 Schleyer, P. von R.; Maerker, C.; Dransfeld, A.; Jiao, H.; van Eikema Hommes, N. J. R., J. Am. Chem. Soc. 1996, 118, 6317-6318. 